\title{
Research on the Dynamics of an Omnidirectional Platform Taking into Account Real Design of Mecanum Wheels (as Exemplified by KUKA youBot)
}

\author{
B. I. Adamov, G. R. Saipulaev
}

The subject of this study is an omnidirectional mobile platform equipped with four Mecanum wheels. The movement of the system on a horizontal plane is considered. The aim of this research is to study the dynamics of the omnidirectional platform, taking into account the design of Mecanum wheels: the shape of the rollers and their finite number. The equations of motion of the onmidirectional mobile platform are derived taking into account the real design of the Mecanum wheels and their slippage. A comparative analysis of the results of numerical modeling for different models of contact friction forces is presented. It has been established that switching of contact rollers and displacement of contact points lead to the occurrence of high-frequency components of wheel rotation speeds, as well as an offset of their average values (in comparison with the modeling results without taking into account the design features of the chassis).

Keywords: omniplatform, Mecanum platform, Mecanum wheel, youBot, omniwheel

\section{Introduction}

The subject of this study is a mobile platform for omnidirectional movement, equipped with Mecanum wheels (Swedish wheels or Ilon wheels [1]). On the periphery of each of them several rollers are mounted, the axes of which skew with the axis of the wheel at an angle of $45^{\circ}$. The design of the Mecanum wheels allows the vehicle to make omnidirectional movement (i.e., in any direction with an arbitrary orientation), for example, to make translation motion along an arbitrary trajectory or to make a turn in place.

Received August 15, 2019

Accepted January 22, 2020

Boris I. Adamov

adamoff.b@yandex.ru

Gasan R. Saipulaev

bot05_00@mail.ru

National Research University "MPEI"

ul. Krasnokazarmennaya 14, Moscow, 111250 Russia 
Due to their mobility and ease of parking, omnidirectional platforms are used to work under the cramped conditions of storage, production and similar premises, and to create vehicles for disabled persons and for other purposes [2].

Mecanum wheels are used to drive spherical wheels [3] and outer shells of spherical robots $[4,5]$.

Mobile omni and Mecanum wheeled platforms are actively studied.

Most theoretical studies of the mechanics of omnidirectional platforms use simplified models of omni and Mecanum wheels. They are modeled by disks, slipping at the contact points with the floor in the direction of the axis of the roller [6-9], or it is assumed that the position of the contact point on the roller does not depend on the angle of rotation of the wheel $[10,11]$. These simplifications allow a description of the dynamics of the system in the framework of nonholonomic mechanics and reduce the dimension of the system of differential equations of motion.

However, it should be noted that some design features of the Mecanum wheels lead to negative effects: the occurrence of vibrations when moving at high speeds and a decrease in the energy efficiency of the control forces (see the review in [12]), which can be studied with an increase in the level of detail of the description of the models of wheels and contact forces.

The results of an experimental study of the dynamics of the controlled movement of the four-wheeled Mecanum platform are given in [13]. It has been established that with sufficiently accurate speed control of wheel speeds, the actual trajectories are significantly different from those required. Also, experiments showed that, with the same initial state of the platform, the same desired motion is tracked with different accuracy. These phenomena are explained by the use of the model, which does not take into account the actual size and number of rollers, their slippage, the dependence of the system's movement on the initial location of the contacting rollers.

In a series of works [14-17], the influence of the real omniwheel design and inertia of the rollers is studied: stability of such systems [15], their computer modeling [16] are considered; tangent impacts occuring due to switching contacting rollers are studied in [17].

A mathematically rigorous description of the geometry of the Mecanum wheels and their kinematics is given in [18]. The influence of the real design of the Mecanum wheels on the accuracy of the odometric navigation of an omnidirectional platform was studied in [19].

In the present paper, the KUKA mobile robotic platform youBot is chosen as a specific object of study [20] (see Fig. 1). The open and free software of this robot allows it to be used for educational and scientific purposes.

To increase the loading ability of the Mecanum wheels of the youBot robot, each of them is equipped with six rather large rollers, the axes of which are equipped with bearings (see Fig. 1). It is expected that the relatively large size of the rollers and their small number will significantly affect the dynamics of the investigated system.

In the present paper, the movement of the youBot Mecanum platform on the horizontal plane (the floor) is investigated taking into account the real geometry of the rollers, linear friction in the joints of the bodies, and wheel slippage.

\section{Description of the Mecanum platform}

In order to describe the kinematics of a mobile robot, we introduce a fixed coordinate frame $x y z$ with a horizontal plane $x y$, and a moving coordinate frame $O X Y Z$ with origin at the geometric center of the platform $O$. The axis $O X$ is a longitudinal symmetry axis of the 


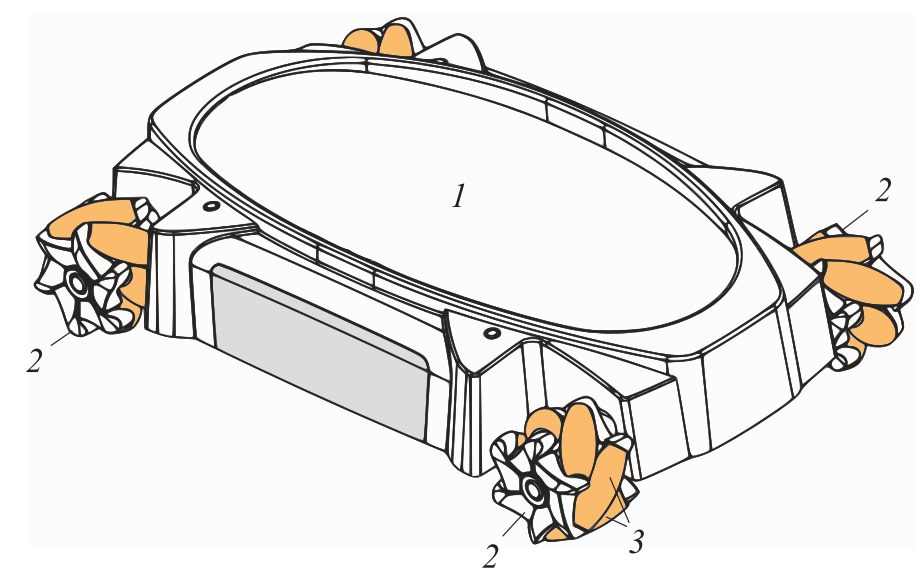

(a) General view

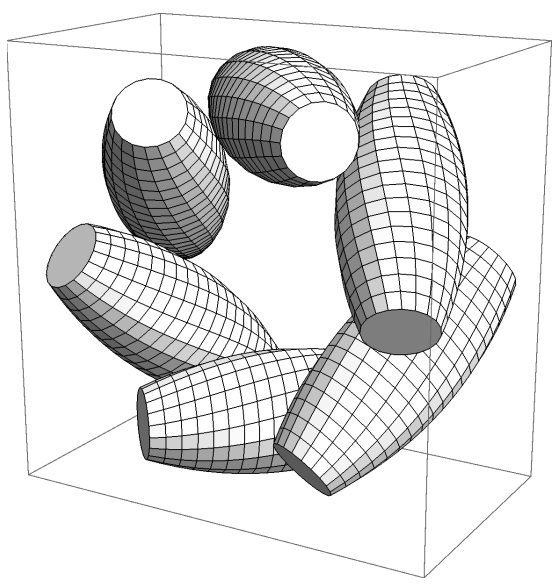

(b) Rollers of one wheel

Fig. 1. Mobile omnidirectional platform of the youBot robot (1 - platform; $2-$ Mecanum wheels; $3-$ rollers)
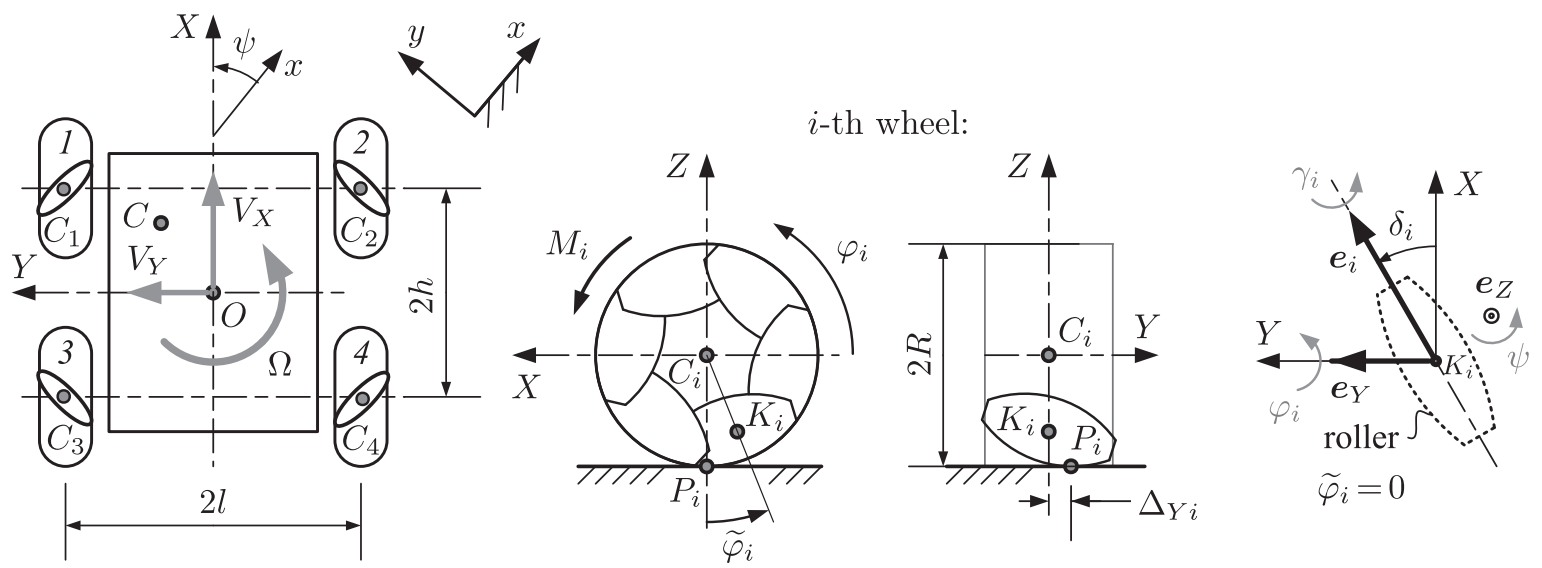

Fig. 2. Kinematic scheme of the mobile platform.

platform, and the $O Y$ axis is a transverse axis. The coordinate axes $z$ and $O Z$ are vertical and aligned with each other. The kinematic scheme of the Mecanum wheeled platform is shown in Fig. 2.

The position of the platform is defined by the Cartesian coordinates $x_{O}$ and $y_{O}$ of its geometric center $O$ in the fixed frame $x y z$ and by the angle $\psi=\angle(x, O X)$.

The platform's center of mass $C$ is displaced relative to the point $O$. Its Cartesian coordinates in the moving plane $O X Y$ are denoted by $a_{X}$ and $a_{Y}$, respectively (see Fig. 2).

The youBot mobile robot is equipped by four pairwise coaxial Mecanum wheels with six peripheral rollers. In the kinematic scheme of the platform, the rollers in contact with the floor are shown as ovals on wheels $1,2,3,4$.

The centers of the wheels $C_{i}, i=\overline{1,4}$, and the platform's center of mass $C$ lie in the $O X Y$ plane. The axes of rotation of the wheels pass through points $C_{i}$ parallel to the $Y$ axis.

The angles of rotation of the $i$ th wheel $(i=\overline{1,4})$ relative the robot base are denoted by $\varphi_{i}$, and the angle of rotation of the contacting roller, by $\gamma_{i}$ (see Fig. 2). 
The angle between the $X Z$ plane and the unit vector $\boldsymbol{e}_{i}$ for the contacting roller axis of the $i$ th wheel is equal to $\delta_{i}$ :

$$
\delta_{i}=\angle\left(X Z, \boldsymbol{e}_{i}\right), \quad\left|\delta_{i}\right|=45^{\circ} .
$$

Also, the value $\delta_{i}$ can be defined as the angle between the $X$ axes and the vector $\boldsymbol{e}_{i}$ when the latter is horizontal $(\widetilde{\varphi}=0$, Fig. 2$)$ :

$$
\delta_{i}=\angle\left(X,\left.\boldsymbol{e}_{i}\right|_{\widetilde{\varphi}=0}\right) .
$$

The Cartesian coordinates of the $i$ th wheel center $C_{i}$ in the $O X Y$ plane are denoted by $\rho_{X i}$ and $\rho_{Y i}$.

In accordance with the kinematic scheme (Fig. 2),

$$
\begin{aligned}
& \delta_{1}=-45^{\circ}, \rho_{X 1}=h, \quad \rho_{Y 1}=l ; \quad \delta_{2}=45^{\circ}, \quad \rho_{X 2}=h, \quad \rho_{Y 2}=-l ; \\
& \delta_{3}=45^{\circ}, \quad \rho_{X 3}=-h, \rho_{Y 3}=l ; \quad \delta_{4}=-45^{\circ}, \rho_{X 4}=-h, \rho_{Y 4}=-l \text {. }
\end{aligned}
$$

All rollers of each wheel contact internally a cylindrical surface of radius $R$, coaxial to the wheel. Let $P_{i}$ be the point of contact of the $i$ th wheel with the floor. Denote by $\Delta_{Y i}$ the displacement of the $P_{i}$ point relative to the wheel center $C_{i}$ in the direction of the $Y$ axis (see Fig. 2). The contact point $P_{i}$ is not displaced along the $X$ axis. The dependence of $\Delta_{Y i}$ on the angle of rotation of the wheel is discussed in the next section.

For convenience of writing, we introduce the symbol $\widetilde{\varphi}_{i}$ to denote the angle of rotation of the contacting roller of the $i$ th wheel about the $Y$ axis (Fig. 2). As each wheel is equipped by six rollers, $\widetilde{\varphi}_{i}$ lies in the range of $-30^{\circ}$ to $30^{\circ}$. If the contact roller switching occurs, $\widetilde{\varphi}_{i}$ jumps from $30^{\circ}$ to $-30^{\circ}$ or from $-30^{\circ}$ to $30^{\circ}$ (depending on the direction of rotation of the wheel).

It is assumed that only one roller of each wheel can contact the floor at any instant.

Thus, the dependence of $\widetilde{\varphi}_{i}$ on the angle of rotation of the $i$ th wheel is given by the formula

$$
\widetilde{\varphi}_{i}=\left(\left(\varphi_{i}+30^{\circ}\right) \bmod 60^{\circ}\right)-30^{\circ}, \quad-30^{\circ} \leqslant \widetilde{\varphi}_{i}<30^{\circ},
$$

where the function $\alpha \bmod 60^{\circ}$ returns the remainder of dividing $\alpha$ by $60^{\circ}$ (the modulo operation).

Further, if necessary, the following brief notation for trigonometric functions is used:

$$
\sin \widetilde{\varphi}_{i}=\mathrm{s} \widetilde{\varphi}_{i}, \quad \cos \widetilde{\varphi}_{i}=\mathrm{c} \widetilde{\varphi}_{i}, \quad \sin \delta_{i}=\mathrm{s} \delta_{i}, \quad \cos \delta_{i}=\mathrm{c} \delta_{i} .
$$

\section{Kinematic model of the Mecanum wheel}

We choose the moving coordinate system $O X Y Z$ as the main one. Further, the coordinate representations of all vectors are given in the $X Y Z$ frame.

We denote by $V_{X}, V_{Y}$ the projections of the velocity vector of the center of the platform $O$ onto the corresponding moving axes, and the angular velocity of the platform, by $\Omega$ :

$$
\begin{aligned}
V_{X} & =\dot{x}_{O} \cos \psi+\dot{y}_{O} \sin \psi, \\
V_{Y} & =-\dot{x}_{O} \sin \psi+\dot{y}_{O} \cos \psi, \\
\Omega & =\dot{\psi} .
\end{aligned}
$$

The velocity vector of the contact point $P_{i}$ of the $i$ th wheel is expressed by the formula

$$
\boldsymbol{v}_{P_{i}}=\boldsymbol{v}_{O}+\Omega \boldsymbol{e}_{Z} \times \boldsymbol{r}_{O P_{i}}+\dot{\varphi}_{i} \boldsymbol{e}_{Y} \times \boldsymbol{r}_{C_{i} P_{i}}+\dot{\gamma}_{i} \boldsymbol{e}_{i} \times \boldsymbol{r}_{K_{i} P_{i}},
$$


where

- $\boldsymbol{v}_{O}=\left(V_{X}, V_{Y}, 0\right)^{\mathrm{T}}$ is the velocity vector of the center of the platform $O$;

- $\boldsymbol{e}_{Y}=(0,1,0)^{\mathrm{T}}, \boldsymbol{e}_{Z}=(0,0,1)^{\mathrm{T}}$ are the unit vectors for the $Y$ and the $Z$ axes;

- $\boldsymbol{e}_{i}=\left(\mathrm{c} \widetilde{\varphi}_{i} \mathrm{c} \delta_{i}, \mathrm{~s} \delta_{i},-\mathrm{s} \widetilde{\varphi}_{i} \mathrm{c} \delta_{i}\right)^{\mathrm{T}}$ is the unit vector for the contacting roller axis;

- the radius vectors are

$$
\begin{gathered}
\boldsymbol{r}_{O P_{i}}=\left(\rho_{X i}, \rho_{Y i},-R\right)^{\mathrm{T}}, \quad \boldsymbol{r}_{C_{i} P_{i}}=\left(0, \Delta_{Y i},-R\right)^{\mathrm{T}}, \\
\boldsymbol{r}_{K_{i} P_{i}}=\boldsymbol{r}_{C_{i} P_{i}}-\boldsymbol{r}_{C_{i} K_{i}}, \quad \boldsymbol{r}_{C_{i} K_{i}}=\left(R_{d} \mathrm{~s} \widetilde{\varphi}_{i}, 0, R_{d} \mathrm{c} \widetilde{\varphi}_{i}\right)^{\mathrm{T}}, \quad R_{d}=\left|C_{i} K_{i}\right| .
\end{gathered}
$$

After the necessary transformations in (3.2), we obtain expressions for the projections of the velocity vector $\boldsymbol{v}_{P_{i}}$ onto the moving axes [19]:

$$
\begin{aligned}
& V_{P_{i} X}=V_{X}-\rho_{Y i} \Omega-\dot{\varphi}_{i} R+\dot{\gamma}_{i} \mathrm{~s} \delta_{i}\left(R_{d} \mathrm{c} \widetilde{\varphi}_{i}-R\right)-\Delta_{Y i}\left(\Omega-\dot{\gamma}_{i} \mathrm{~s} \widetilde{\varphi}_{i} \mathrm{c} \delta_{i}\right), \\
& V_{P_{i} Y}=V_{Y}+\rho_{X i} \Omega+\dot{\gamma}_{i} \mathrm{c} \delta_{i}\left(R \mathrm{c} \widetilde{\varphi}_{i}-R_{d}\right), \\
& V_{P_{i} Z}=\dot{\gamma}_{i}\left(\Delta_{Y i} \mathrm{c} \widetilde{\varphi}_{i} \mathrm{c} \delta_{i}-R_{d} \mathrm{~s} \widetilde{\varphi}_{i} \mathrm{~s} \delta_{i}\right) .
\end{aligned}
$$

From the assumption of motion of the contacting rollers without bouncing $\left(V_{P_{i} Z} \equiv 0\right)$, we find the dependence of the displacements of the contact points, $\Delta_{Y i}$, on angles $\widetilde{\varphi}_{i}$ :

$$
\Delta_{Y i}=R_{d} \tan \widetilde{\varphi}_{i} \tan \delta_{i} .
$$

This relationship for $\Delta_{Y i}$ is equivalent to that obtained by A. Gfrerrer by methods of descriptive geometry [18].

\section{Dynamical model of the system taking into account the design of the wheels and slippage of the contact points}

Derive the equations of motion of the system under following assumptions:

- the rollers, as well as parts of mechanical gears of the wheel drives, are weightless;

- the linear velocity friction forces take place in joints of bodies;

- the contact of the rollers with the floor is a point, slippage takes place.

We describe the dynamics of the system in quasi-velocities $V_{X}, V_{Y}, \Omega, \dot{\varphi}_{i}, \dot{\gamma}_{i}(i=\overline{1,4})$ using Appell's equations [21-23]

$$
\frac{\partial S}{\partial \dot{V}_{X}}=\Pi_{V_{X}}, \quad \frac{\partial S}{\partial \dot{V}_{Y}}=\Pi_{V_{Y}}, \quad \frac{\partial S}{\partial \dot{\Omega}}=\Pi_{\Omega}, \quad \frac{\partial S}{\partial \ddot{\varphi}_{i}}=\Pi_{\varphi_{i}}, \quad \frac{\partial S}{\partial \ddot{\gamma}_{i}}=\Pi_{\gamma_{i}}
$$

where $S$ is the energy of accelerations of the system, and $\Pi_{V_{X}}, \Pi_{V_{Y}}, \Pi_{\Omega}, \Pi_{\varphi_{i}}, \Pi_{\gamma_{i}}$ are generalized forces associated with quasi-velocities.

\subsection{Inertial terms of the equations of motion}

In the problem considered

$$
S=S_{p}+\sum_{i=1}^{4} S_{i},
$$

where $S_{p}$ and $S_{i}$ are the energies of accelerations of the platform and of the $i$ th wheel, respectively. 
The acceleration energy of the platform has the form [21-23]:

$$
S_{p}=\frac{1}{2}\left\{m\left(w_{C X}^{2}+w_{C Y}^{2}\right)+I_{C} \dot{\Omega}^{2}\right\},
$$

where $w_{C X}=\dot{V}_{X}-a_{Y} \dot{\Omega}-\Omega\left(V_{Y}+a_{X} \Omega\right)$ and $w_{C Y}=\dot{V}_{Y}+a_{X} \dot{\Omega}+\Omega\left(V_{X}-a_{Y} \Omega\right)$ are the components of the acceleration of the platform's center of mass $C, m$ and $I_{C}$ are its mass and inertia moment relative to the axis $C Z ; a_{X}$ and $a_{Y}$ are the coordinates of the center of mass $C$ in the plane $O X Y$. Here and in the sequel, in the expressions for energies of accelerations, we omit terms independent of quasi-acceleration.

Assuming that the center of mass of the $i$ th wheel is at the geometric center $C_{i}$, and $C_{i} Y$ is the axis of dynamical symmetry, the expression for $S_{i}$ takes the form [22-24]

$$
S_{i}=\frac{1}{2}\left\{m_{1} \boldsymbol{w}_{C_{i}}^{2}+I_{1} \dot{\Omega}^{2}+J_{1} \ddot{\varphi}_{i}^{2}\right\},
$$

where $m_{1}$ is the mass of the wheel, $I_{1}$ and $J_{1}$ are its inertia moments relative to the axes $C_{i} Z$ and $C_{i} Y$, respectively, and $\boldsymbol{w}_{C_{i}}$ is the acceleration of the center of mass $C_{i}$.

The total energy of accelerations of the four-wheeled system is

$$
\begin{aligned}
\sum S_{i}=\frac{1}{2}\left\{m_{1} \sum \boldsymbol{w}_{C_{i}}^{2}+4 I_{1} \dot{\Omega}^{2}+J_{1} \sum \ddot{\varphi}_{i}^{2}\right\}= \\
=\frac{1}{2}\left\{4 m_{1}\left(w_{O X}^{2}+w_{O Y}^{2}\right)+4 m_{1}\left(l^{2}+h^{2}\right) \dot{\Omega}^{2}+4 I_{1} \dot{\Omega}^{2}+J_{1} \sum \ddot{\varphi}_{i}^{2}\right\},
\end{aligned}
$$

where $w_{O X}=\dot{V}_{X}-\Omega V_{Y}$ and $w_{O Y}=\dot{V}_{Y}+\Omega V_{X}$ are the acceleration components of the point $O$, which is the center of mass of the wheeled system.

In accordance with (4.2)-(4.4), the inertial terms in Appell's equations (4.1) take the form

$$
\begin{aligned}
\frac{\partial S}{\partial \dot{V}_{X}} & =m_{s}\left(\dot{V}_{X}-\Omega V_{Y}\right)-m\left(a_{Y} \dot{\Omega}+a_{X} \Omega^{2}\right), \\
\frac{\partial S}{\partial \dot{V}_{Y}} & =m_{s}\left(\dot{V}_{Y}+\Omega V_{X}\right)+m\left(a_{X} \dot{\Omega}-a_{Y} \Omega^{2}\right), \\
\frac{\partial S}{\partial \dot{\Omega}} & =I_{s} \dot{\Omega}+m a_{X}\left(\dot{V}_{Y}+\Omega V_{X}\right)-m a_{Y}\left(\dot{V}_{X}-\Omega V_{Y}\right), \\
\frac{\partial S}{\partial \ddot{\varphi}_{i}} & =J_{1} \ddot{\varphi}_{i}, \quad \frac{\partial S}{\partial \ddot{\gamma}_{i}}=0, \quad i=\overline{1,4},
\end{aligned}
$$

where $m_{s}=m+4 m_{1}$ is the total mass of the robot and $I_{s}=4 I_{1}+4 m_{1}\left(h^{2}+l^{2}\right)+I_{C}+m\left(a_{X}^{2}+a_{Y}^{2}\right)$ is its moment of inertia relative to the axis $O Z$.

\subsection{Generalized forces}

It is assumed that the movement of the system occurs under the action of the following active forces:

- actuator torques $M_{i}$;

- linear viscous friction torques in the wheel-base joints:

$$
M_{\mathrm{fw} i}=-\mu_{1} \dot{\varphi}_{i}
$$

where $\mu_{1}$ is the proportionality coefficient; 
- linear viscous friction torques in the wheel-roller joints:

$$
M_{\mathrm{fr} i}=-\mu_{2} \dot{\gamma}_{i},
$$

where $\mu_{2}$ is the proportionality coefficient;

- friction forces at the contact points of the rollers with the floor:

$$
\boldsymbol{F}_{i}=\left(F_{X i}, F_{Y i}, 0\right)^{\mathrm{T}} .
$$

Define the generalized forces in Appell's equations using the condition of balance of virtual powers

$$
\begin{aligned}
& \sum_{i=1}^{4}\left(F_{X i} V_{P_{i} X}^{\mathfrak{v}}+F_{Y i} V_{P_{i} Y}^{\mathfrak{v}}+\left(M_{i}+M_{\mathrm{fw} i}\right) \dot{\varphi}_{i}^{\mathfrak{v}}+M_{\mathrm{fr} i} \dot{\gamma}_{i}^{\mathfrak{v}}\right)= \\
& =\Pi_{V_{X}} V_{X}^{\mathfrak{v}}+\Pi_{V_{Y}} V_{Y}^{\mathfrak{v}}+\Pi_{\Omega} \Omega^{\mathfrak{v}}+\sum_{i=1}^{4}\left(\Pi_{\varphi_{i}} \dot{\varphi}_{i}^{\mathfrak{v}}+\Pi_{\gamma_{i}} \dot{\gamma}_{i}^{\mathfrak{v}}\right)
\end{aligned}
$$

where $\mathfrak{v}$ denotes virtual velocities [24], for which, due to stationarity of the constraints and formulas (3.3), the following relations take place:

$$
\begin{aligned}
& V_{P_{i} X}^{\mathfrak{v}}=V_{X}^{\mathfrak{v}}-\rho_{Y i} \Omega^{\mathfrak{v}}-R \dot{\varphi}_{i}^{\mathfrak{v}}+\dot{\gamma}_{i}^{\mathfrak{v}} \mathrm{s} \delta_{i}\left(R_{d} \mathrm{c} \widetilde{\varphi}_{i}-R\right)-\Delta_{Y i}\left(\Omega^{\mathfrak{v}}-\dot{\gamma}_{i}^{\mathfrak{v}} \mathrm{s} \widetilde{\varphi}_{i} \mathrm{c} \delta_{i}\right), \\
& V_{P_{i} Y}^{\mathfrak{v}}=V_{Y}^{\mathfrak{v}}+\rho_{X i} \Omega^{\mathfrak{v}}+\dot{\gamma}_{i}^{\mathfrak{v}} \mathrm{c} \delta_{i}\left(R \mathrm{c} \widetilde{\varphi}_{i}-R_{d}\right), \quad i=\overline{1,4} .
\end{aligned}
$$

Substituting the last expressions into the power balance equation gives the following expressions for the generalized forces:

$$
\begin{aligned}
& \Pi_{V_{X}}=\sum_{i} F_{X i}, \quad \Pi_{V_{Y}}=\sum_{i} F_{Y i}, \quad \Pi_{\Omega}=\sum_{i}\left[F_{Y i} \rho_{X i}-F_{Y i}\left(\rho_{Y i}+\Delta_{Y i}\right)\right], \\
& \Pi_{\gamma_{i}}=-\mu_{2} \dot{\gamma}_{i}-F_{X i}\left[-\Delta_{Y i} \mathrm{~s} \widetilde{\varphi}_{i} \mathrm{c} \delta_{i}+\mathrm{s} \delta_{i}\left(R-R_{d} \mathrm{c} \widetilde{\varphi}_{i}\right)\right]-F_{Y i} \mathrm{c} \delta_{i}\left(R_{d}-R \mathrm{c} \widetilde{\varphi}_{i}\right), \\
& \Pi_{\varphi_{i}}=M_{i}-\mu_{1} \dot{\varphi}_{i}-F_{X i} R, \quad i=\overline{1,4} .
\end{aligned}
$$

\subsection{Equations of motion}

Write down Appell's equations (4.1) taking into account Eqs. (4.5) and (4.8):

$$
\begin{aligned}
& m_{s}\left(\dot{V}_{X}-\Omega V_{Y}\right)-m\left(a_{Y} \dot{\Omega}+a_{X} \Omega^{2}\right)=\sum F_{X i} \\
& m_{s}\left(\dot{V}_{Y}+\Omega V_{X}\right)+m\left(a_{X} \dot{\Omega}-a_{Y} \Omega^{2}\right)=\sum F_{Y i}, \\
& I_{s} \dot{\Omega}+m a_{X}\left(\dot{V}_{Y}+\Omega V_{X}\right)-m a_{Y}\left(\dot{V}_{X}-\Omega V_{Y}\right)=\sum\left[F_{Y i} \rho_{X i}-F_{X i}\left(\rho_{Y i}+\Delta_{Y i}\right)\right] \\
& J_{1} \ddot{\varphi}_{i}=M_{i}-\mu_{1} \dot{\varphi}_{i}-F_{X i} R, \\
& 0=-\mu_{2} \dot{\gamma}_{i}-F_{X i}\left[\mathrm{~s} \delta_{i}\left(R-R_{d} \mathrm{c} \widetilde{\varphi}_{i}\right)-\Delta_{Y i} \mathrm{~s} \widetilde{\varphi}_{i} \mathrm{c} \delta_{i}\right]-F_{Y i} \mathrm{c} \delta_{i}\left(R_{d}-R \mathrm{c} \widetilde{\varphi}_{i}\right), \quad i=\overline{1,4}
\end{aligned}
$$

This system of equations is closed by relations (3.4) for displacements $\Delta_{Y i}$ and a contact force model $F_{X i}, F_{Y i}$.

In this paper we consider the following contact friction models:

- the linear friction model at the contact points $P_{i}$ :

$$
F_{X i}=-\mu_{0} V_{P_{i} X}, \quad F_{Y i}=-\mu_{0} V_{P_{i} Y},
$$

where $\mu_{0}$ is a "big" coefficient; 
- the Coulomb friction model at the contact points $P_{i}$ :

$$
F_{X i}=-f N_{i} \frac{V_{P_{i} X}}{\sqrt{V_{P_{i} X}^{2}+V_{P_{i} Y}^{2}}}, \quad F_{Y i}=-f N_{i} \frac{V_{P_{i} Y}}{\sqrt{V_{P_{i} X}^{2}+V_{P_{i} Y}^{2}}},
$$

where $f$ is the coefficient of sliding friction and $N_{i}$ is the normal support reaction.

In the latter case, finding normal reactions $N_{i}$ (Fig. 3) is required.
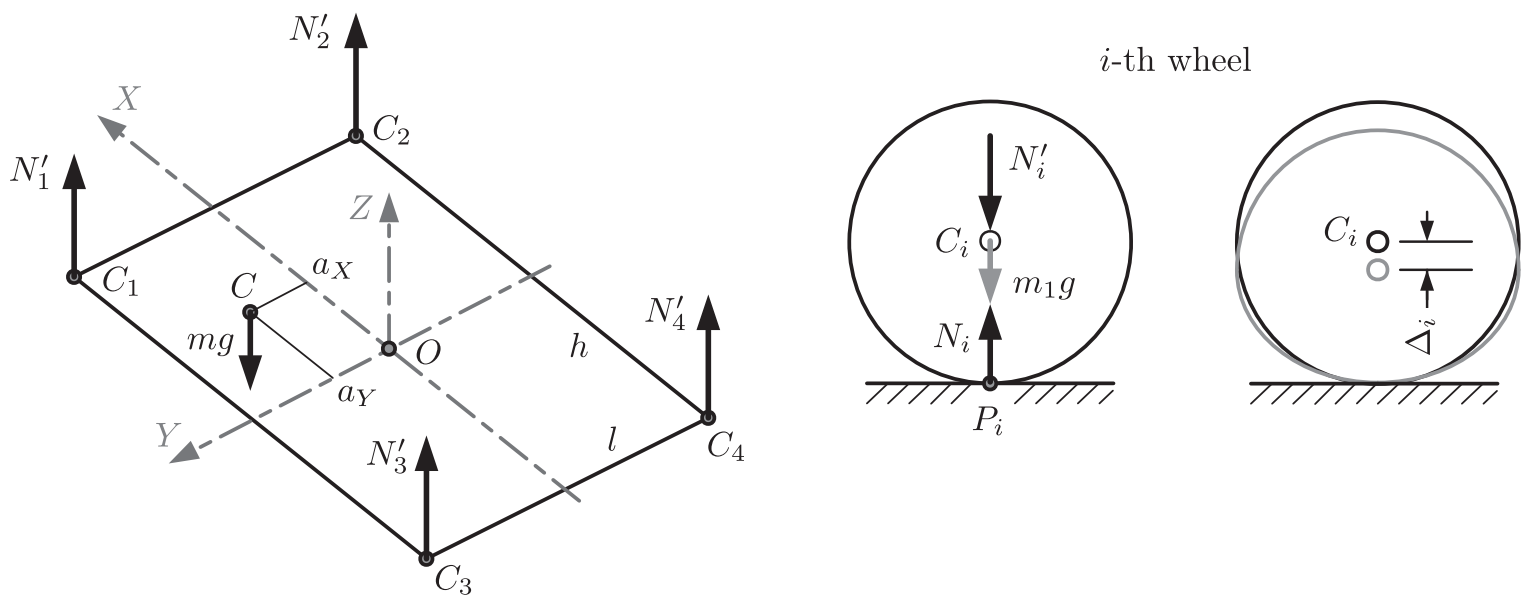

Fig. 3. Scheme for calculation of normal reactions $N_{i}$ (only vertical forces are shown; $N_{i}^{\prime}=N_{i}-m_{1} g$ is the $i$ th joint reaction).

This problem is statically indefinable. To solve it, we accept the hypothesis of finite stiffness of the structural elements of the wheels (Fig. 3). We assume that the normal reactions and the small vertical displacements of the wheel centers $\Delta_{i}$ are related by

$$
N_{i}=-k \Delta_{i}
$$

where $k$ is the stiffness coefficient.

Since the center of mass of the platform $C$ and the wheel centers of mass $C_{i}$ lie in the same plane, the desired normal reactions $N_{i}$ can be found from the equations of statics (Fig. 3):

- the equation of projections of forces on the $Z$ axis

$$
N_{1}+N_{2}+N_{3}+N_{4}-m g-4 m_{1} g=0,
$$

where $g$ is the gravity acceleration;

- the equation of moments of forces with respect to the axis $O X$

$$
\left(N_{1}+N_{3}\right) l-\left(N_{2}+N_{4}\right) l-m g a_{Y}=0 ;
$$

- the equation of moments of forces with respect to the axis $O Y$

$$
\left(N_{3}+N_{4}\right) h-\left(N_{1}+N_{2}\right) h+m g a_{X}=0 ;
$$

- the equation of compatibility of deformations

$$
\frac{\Delta_{1}+\Delta_{4}}{2}=\frac{\Delta_{2}+\Delta_{3}}{2}
$$


The last relation expresses the equality of the displacements of the point $O$ due to the vertical displacement of the centers of the crosswise lying wheels. When deriving this equation, it was assumed that $\Delta_{Y i}=0$.

Solving the system of static equations (4.12)-(4.16), we get

$$
\begin{array}{ll}
N_{1}=m_{1} g+\frac{m g}{4}\left(1+\frac{a_{X}}{h}+\frac{a_{Y}}{l}\right), & N_{2}=m_{1} g+\frac{m g}{4}\left(1+\frac{a_{X}}{h}-\frac{a_{Y}}{l}\right), \\
N_{3}=m_{1} g+\frac{m g}{4}\left(1-\frac{a_{X}}{h}+\frac{a_{Y}}{l}\right), & N_{4}=m_{1} g+\frac{m g}{4}\left(1-\frac{a_{X}}{h}-\frac{a_{Y}}{l}\right) .
\end{array}
$$

Note that if the contact points $P_{i}$ are located asymmetrically relative to the center of the $O$ platform (for $\Delta_{Y i} \neq 0$ ), the expressions of the support reactions $N_{i}$ are significantly complicated.

\section{Platform motion modeling}

\subsection{The models of the system}

We analyze the effect of the real design of Mecanum wheels on the movement of the platform by comparing the results of the numerical solution of the equations of motion for the following models of the system:

- Model 1 is nonholonomic. The following simplifications are used: at each instant, the contact points $P_{i}$ lie on the central cross-sections of the rollers $\left(\widetilde{\varphi}_{i} \equiv 0, \Delta_{Y i} \equiv 0\right)$, there is no slippage $\left(V_{P_{i} X} \equiv 0, V_{P_{i} Y} \equiv 0\right)$. This model is described by the system of equations [10]

$$
\begin{gathered}
\left(m_{s}+\frac{4 J_{1}}{R^{2}}\right) \dot{V}_{X}-m_{s} \Omega V_{Y}-m a_{Y} \dot{\Omega}-m a_{X} \Omega^{2}+\zeta_{X} V_{X}=\frac{M_{X}}{R}, \\
\left(m_{s}+\frac{4 J_{1}}{R^{2}}\right) \dot{V}_{Y}+m_{s} \Omega V_{X}+m a_{X} \dot{\Omega}-m a_{Y} \Omega^{2}+\zeta_{Y} V_{Y}=\frac{M_{Y}}{R}, \\
\left(I_{s}+\frac{4(l+h)^{2}}{R^{2}} J_{1}\right) \dot{\Omega}-m a_{Y}\left(\dot{V}_{X}-\Omega V_{Y}\right)+m a_{X}\left(\dot{V}_{Y}+\Omega V_{X}\right)+\mu_{\Omega} \Omega=M_{\Omega}, \\
\dot{\varphi}_{i}=\frac{1}{R}\left[V_{X}+V_{Y} \tan \delta_{i}+\left(\rho_{X i} \tan \delta_{i}-\rho_{Y i}\right) \Omega\right], \quad \dot{\gamma}_{i}=\frac{V_{Y}+\Omega \rho_{X i}}{\left(R_{d}-R\right) \cos \delta_{i}}, \quad i=\overline{1,4}
\end{gathered}
$$

where $\zeta_{X}=\frac{4 \mu_{1}}{R^{2}}, \zeta_{Y}=\frac{4 \mu_{1}}{R^{2}}+\frac{8 \mu_{2}}{\left(R-R_{d}\right)^{2}}, \mu_{\Omega}=\frac{4 \mu_{1}(l+h)^{2}}{R^{2}}+\frac{8 \mu_{2} h^{2}}{\left(R-R_{d}\right)^{2}}$,

$$
\begin{gathered}
M_{X}=M_{2}+M_{1}+M_{4}+M_{3}, \\
M_{Y}=M_{2}-M_{1}-M_{4}+M_{3}, \\
M_{\Omega}=\frac{l+h}{R}\left(M_{2}-M_{1}+M_{4}-M_{3}\right) .
\end{gathered}
$$

Here the contact forces (reactions of the nonholonomic constraints $V_{P_{i} X}=V_{P_{i} Y}=0$, $i=\overline{1,4}$ ) are unlimited.

- Model 2 is given by the dynamics equations (4.9) with the following contact forces:

- linear friction (4.10) (Model 2a) or

- Coulomb friction (4.11), (4.17) (Model 2b). 
Note that model $\mathbf{2} \mathbf{a}$ is an approximation of the nonholonomic model of the system: if the coefficient of viscous friction $\mu_{0}$ increases, the slippage at the contact points of the rollers decreases.

In this article, the strict no-slip condition is not considered: due to the discontinuous nature of the dependence $\widetilde{\varphi}_{i}\left(\varphi_{i}\right)$, the conditions $V_{P i X}=V_{P i Y}=0$ assume the presence of impacts when changing the contact rollers, which complicates the dynamic analysis of the system.

\subsection{Input data for modeling}

Initial conditions for variables and control torques $M_{i}, i=\overline{1,4}$, are chosen to be the same for all three models under consideration using the following algorithm:

1. We set the parameters of the desired platform motion

$$
V_{X}=V_{X}^{*}(t), V_{Y}=V_{Y}^{*}(t), \Omega=\Omega^{*}(t) .
$$

2. By substituting $V_{X}=V_{X}^{*}(t), V_{Y}=V_{Y}^{*}(t), \Omega=\Omega^{*}(t)$ in the equations for model $\mathbf{1}$, the dependencies $M_{X}=M_{X}^{*}(t), M_{Y}=M_{Y}^{*}(t), M_{\Omega}=M_{\Omega}^{*}(t), \dot{\varphi}_{i}=\dot{\varphi}_{i}^{*}(t), \dot{\gamma}_{i}=\dot{\gamma}_{i}^{*}(t), i=\overline{1,4}$, are determined.

3. The input control torques on the desired motion are calculated using the following formulas [10]:

$$
\begin{aligned}
& M_{1}^{*}(t)=\frac{1}{4}\left\{M_{X}^{*}(t)-M_{Y}^{*}(t)-\frac{R}{h+l} M_{\Omega}^{*}(t)\right\}, M_{2}^{*}(t)=\frac{1}{4}\left\{M_{X}^{*}(t)+M_{Y}^{*}(t)+\frac{R}{h+l} M_{\Omega}^{*}(t)\right\}, \\
& M_{3}^{*}(t)=\frac{1}{4}\left\{M_{X}^{*}(t)+M_{Y}^{*}(t)-\frac{R}{h+l} M_{\Omega}^{*}(t)\right\}, M_{4}^{*}(t)=\frac{1}{4}\left\{M_{X}^{*}(t)-M_{Y}^{*}(t)+\frac{R}{h+l} M_{\Omega}^{*}(t)\right\} .
\end{aligned}
$$

4. The found torque change laws $M_{i}=M_{i}^{*}(t), i=\overline{1,4}$, as well as initial conditions $V_{X}(0)=V_{X}^{*}(0), V_{Y}(0)=V_{Y}^{*}(0), \Omega(0)=\Omega^{*}(0), \dot{\varphi}(0)=\dot{\varphi}_{i}^{*}(0), \dot{\gamma}_{i}(0)=\dot{\gamma}_{0}^{*}(0)$ are used for models $2 \mathbf{a}$ and $\mathbf{2 b}$.

The results of mathematical modeling with the described method for calculating the input torques and choosing the initial conditions illustrate systematic errors of open-loop control that occur due to ignoring some features of the wheel design and contact forces limitations.

Note that the use of open-loop control $\left(M_{i}=M_{i}^{*}(t)\right)$, without feedbacks on speeds and coordinates, allows one to exclude the influence of transients in the control loop on the movement of the system.

In order to eliminate nonuniqueness of the solution and ensure correct application of schemes for numerical solution of differential equations, regularization of the equations for model 2 is carried out:

- Under the sign of the radical in the calculation formulas (4.11) for Coulomb contact forces, a small term $\nu^{2}$ is introduced, eliminating uncertainty when $V_{P_{i} X}=V_{P_{i} Y}=0$ :

$$
F_{X i} \approx-f N_{i} \frac{V_{P_{i} X}}{\sqrt{\nu^{2}+V_{P_{i} X}^{2}+V_{P_{i} Y}^{2}}}, \quad F_{Y i} \approx-f N_{i} \frac{V_{P_{i} Y}}{\sqrt{\nu^{2}+V_{P_{i} X}^{2}+V_{P_{i} Y}^{2}}} .
$$

- The right-hand sides of the equations for generalized coordinates $\gamma_{i}$ in (4.9) are assumed to be $J_{2} \dot{\gamma}_{i}$, not 0 . Here $J_{2}>0$ is a small parameter, the introduction of which also allows us to take into account the inertia of the contacting rollers in some approximation. 
Set the following values of the system parameters [20, 25]:

$$
\begin{array}{llll}
m=30.0 \mathrm{~kg}, & m_{1}=1.4 \mathrm{~kg}, & I_{s}=4.42 \mathrm{~kg} \cdot \mathrm{m}^{2}, & J_{1}=5.87 \cdot 10^{-3} \mathrm{~kg} \cdot \mathrm{m}^{2}, \\
a_{X}=1.6 \mathrm{~cm}, & a_{Y}=-2.6 \mathrm{~cm}, & \mu_{1}=0.11 \mathrm{~N} \cdot \mathrm{m} \cdot \mathrm{s}, & \mu_{2}=5.78 \cdot 10^{-3} \mathrm{~N} \cdot \mathrm{m} \cdot \mathrm{s}, \\
l=15.0 \mathrm{~cm}, & h=23.5 \mathrm{~cm}, & R=5.0 \mathrm{~cm}, & R_{d}=3.55 \mathrm{~cm} .
\end{array}
$$

For the coefficient of friction of rollers with the floor, we assume the value $f=0.5$. This value corresponds to the case of movement of rubber rollers on dry asphalt [26].

The values of the regularizing small parameters are

$$
\nu=10^{-3} \mathrm{~m} / \mathrm{s}, \quad J_{2}=10^{-6} \mathrm{~kg} \cdot \mathrm{m}^{2} .
$$

The coefficient of viscous contact friction for model $\mathbf{2 a}$ is selected from the condition of equality of linear and regularized Coulomb contact forces (at the average value of the normal reaction $\left.\left\langle N_{i}\right\rangle=m_{s} g / 4\right)$ in a small neighborhood of a point $V_{X}=V_{Y}=0$ :

$$
\mu_{0}=\frac{f}{\nu} \cdot \frac{m_{s} g}{4}=4.4 \cdot 10^{4} \mathrm{~N} \cdot \mathrm{s} / \mathrm{m}
$$

\subsection{Case 1: Translational motion in a circle}

Consider the uniform translational motion of the platform in a circle as the desired motion:

$$
V_{X}^{*}(t)=-\varrho \omega \cos \left(\omega t-\psi_{0}\right), \quad V_{Y}^{*}(t)=\varrho \omega \sin \left(\omega t-\psi_{0}\right), \quad \Omega^{*}(t)=0,
$$

where $\varrho=2.5 \mathrm{~m}$ is the radius of the circle, $\omega=2 \pi / T$ is the cyclic frequency, $T=30 \mathrm{~s}$ is the period of motion, and $\psi_{0}=90^{\circ}$ is the constant angle of rotation of the platform $\left(\psi(t)=\psi_{0}\right)$.

At the initial time, the wheel angles are $\varphi_{i}(0)=0.1(i=\overline{1,4})$.

The control torques during such a movement change with the frequency $\omega$ according to sinusoidal laws (see Fig. 4).

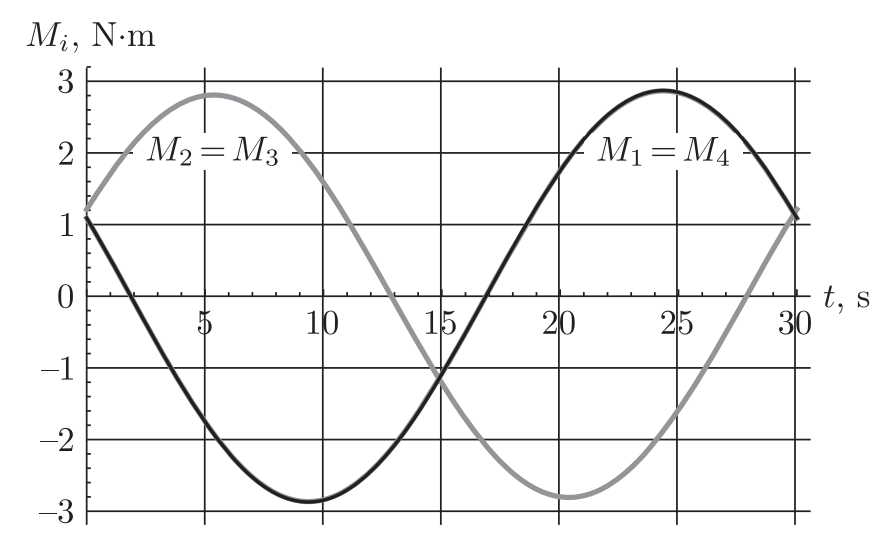

Fig. 4. Control torques for Case 1.

The trajectories of the platform's center $O$ and the rotation angles $\psi$ for three different models of the system are shown in Fig. 5 (traversing counterclockwise). Note that the design features of the Mecanum wheels, which are not taken into account in model 1, lead to a deformation of the trajectory in the direction of the transverse axis $Y$, as well as to the occurrence of angular movements. 

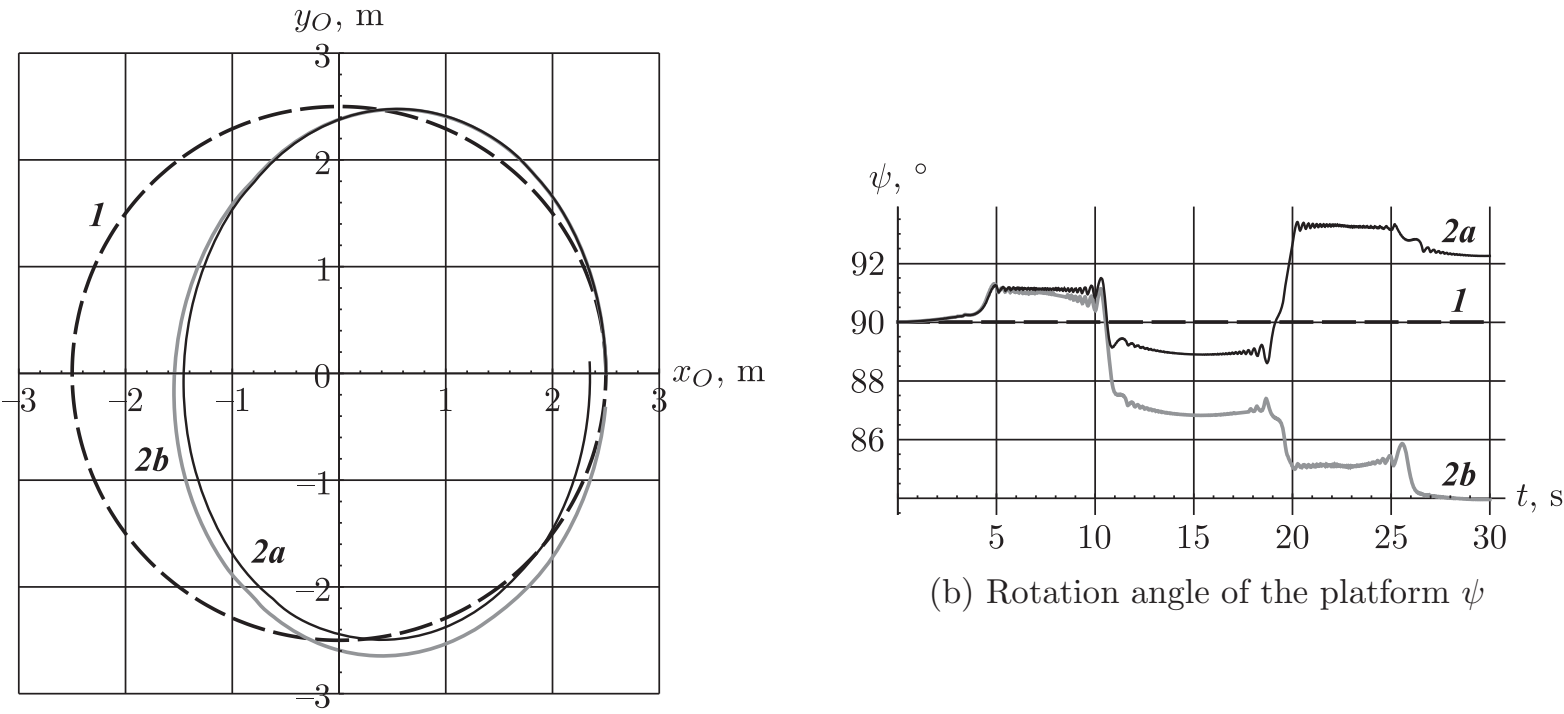

(b) Rotation angle of the platform $\psi$

(a) Trajectories of the center of the platform $O$

Fig. 5. Results of the numerical modeling for Case 1 (the numbers of the curves correspond to those of the models).

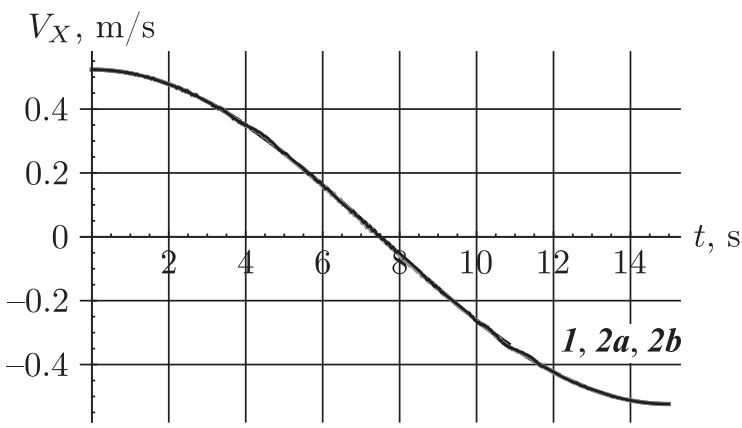

(a) Dependencies $V_{X}(t)$

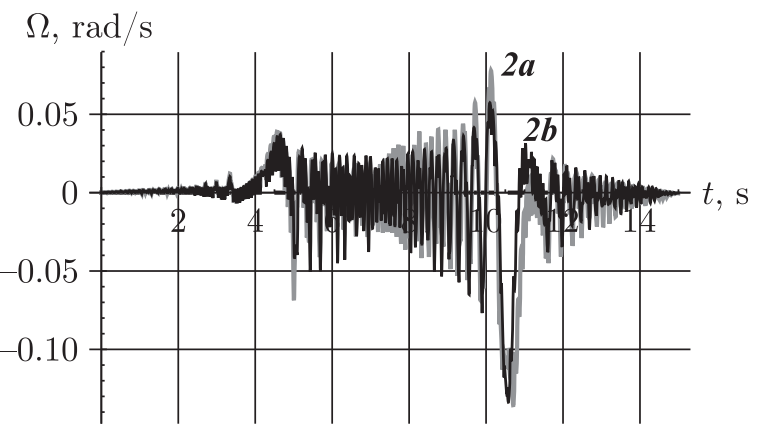

(c) Dependencies $\Omega(t)$

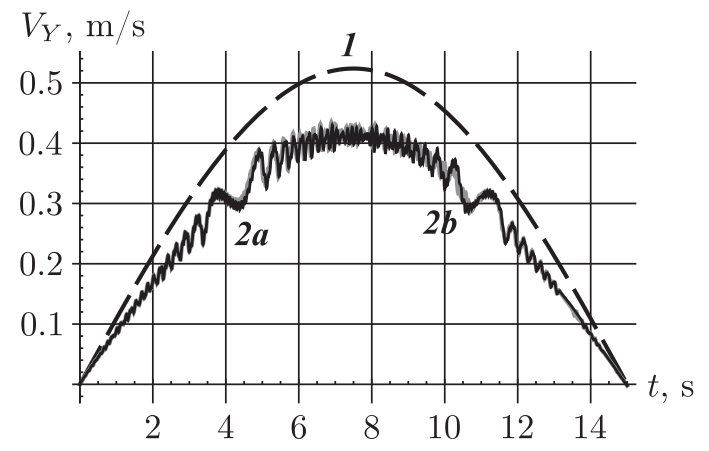

(b) Dependencies $V_{Y}(t)$

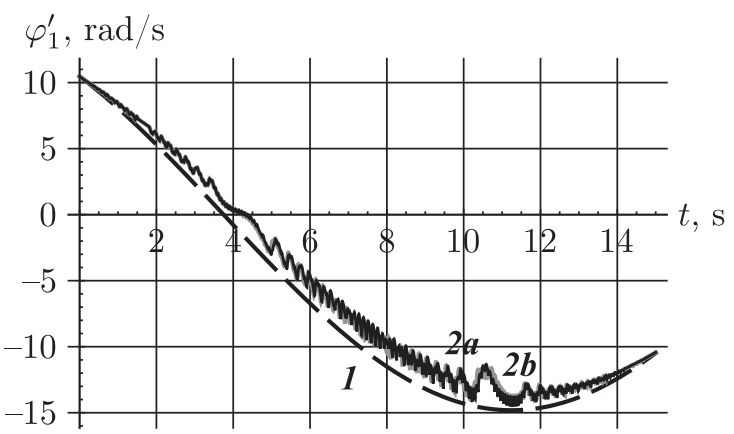

(d) Dependencies $\dot{\varphi}_{1}(t)$

Fig. 6. Results of the numerical modeling for Case 1 (the numbers of the curves correspond to those of the models). 
Figure 6 shows graphs of changes in the platform velocities and, as an example, the rotation speed of the first wheel $(0 \leqslant t \leqslant T / 2)$. They illustrate the occurrence of vibrations due to discontinuous changes in the positions of the contact points $P_{i}$ when switching rollers. In a particular case, according to models $\mathbf{2 a}$ and $\mathbf{2} \mathbf{b}$, the average values of the velocity $V_{Y}$ are less than in accordance with model 1. This demonstrates a decrease in the efficiency of the platform's lateral movement control due to the design features of the Mecanum wheels.

Figure 7 shows graphs of the trajectory of the center $O$ of the platform and the rotation angles $\psi$ for model $\mathbf{2} \mathbf{b}$ with various combinations of initial values of the wheel rotation angles $\varphi_{i}(0)$. In a particular case, at the end of the movement $(t=T)$, the spread of the coordinates of the point $O$ is about $0.6 \mathrm{~m}$, and the spread of the $\psi$ angles is about $15^{\circ}$.
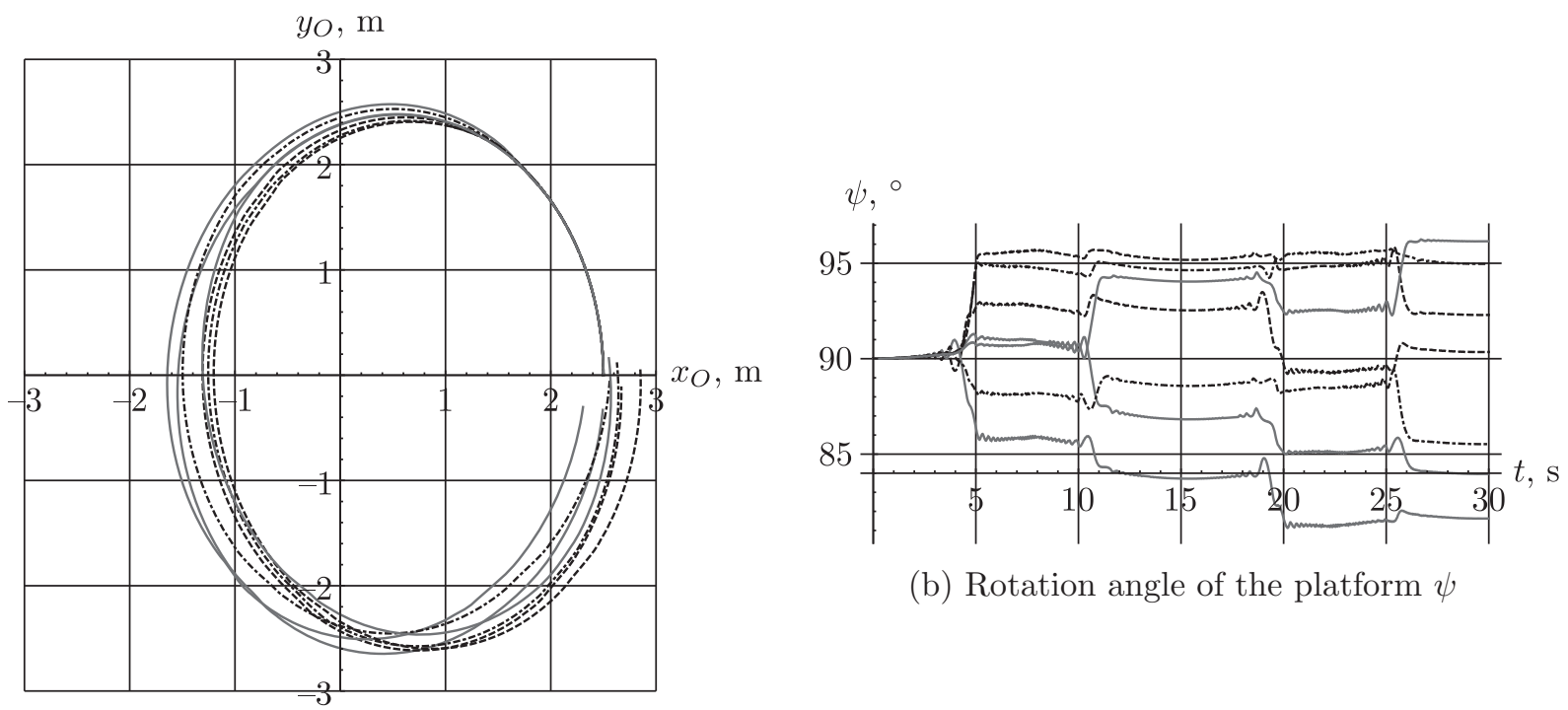

(b) Rotation angle of the platform $\psi$

(a) Trajectories of the center of the platform $O$

Fig. 7. Dependence of the platform's motion on the initial angles of rotation of the wheels.

\subsection{Case 2: Lateral motion around a circle}

Consider the following desired motion: the center $O$ of the platform uniformly moves around a circle of radius $\varrho$ with cyclic frequency $\omega$, and the transverse axis $O Y$ is directed tangentially to the trajectory:

$$
V_{X}^{*}(t)=0, \quad V_{Y}^{*}(t)=\varrho \omega, \quad \Omega^{*}(t)=\omega,
$$

where $\varrho=2.5 \mathrm{~m} ; \omega=2 \pi / T, T=30 \mathrm{~s}$ is the period of motion.

At the initial time, the rotation angle of the platform is $\psi(0)=0$, and the wheel angles are $\varphi_{i}(0)=0.1(i=\overline{1,4})$.

According to model 1, the control torques during such a movement are constant.

The trajectories of the platform's center $O$ and the rotation angles $\psi$ for three different models of the system are shown in Fig. 8 (traversing counterclockwise). Note that the design features of the Mecanum wheels, which are not taken into account in model 1, lead to a decrease in the radius of the trajectory, as well as a decrease in the speed of its traversing.

Figure 9 shows graphs of changes in the platform velocities and of the rotation speed of the first wheel for the initial phase of movement. They illustrate the occurrence of vibrations due 

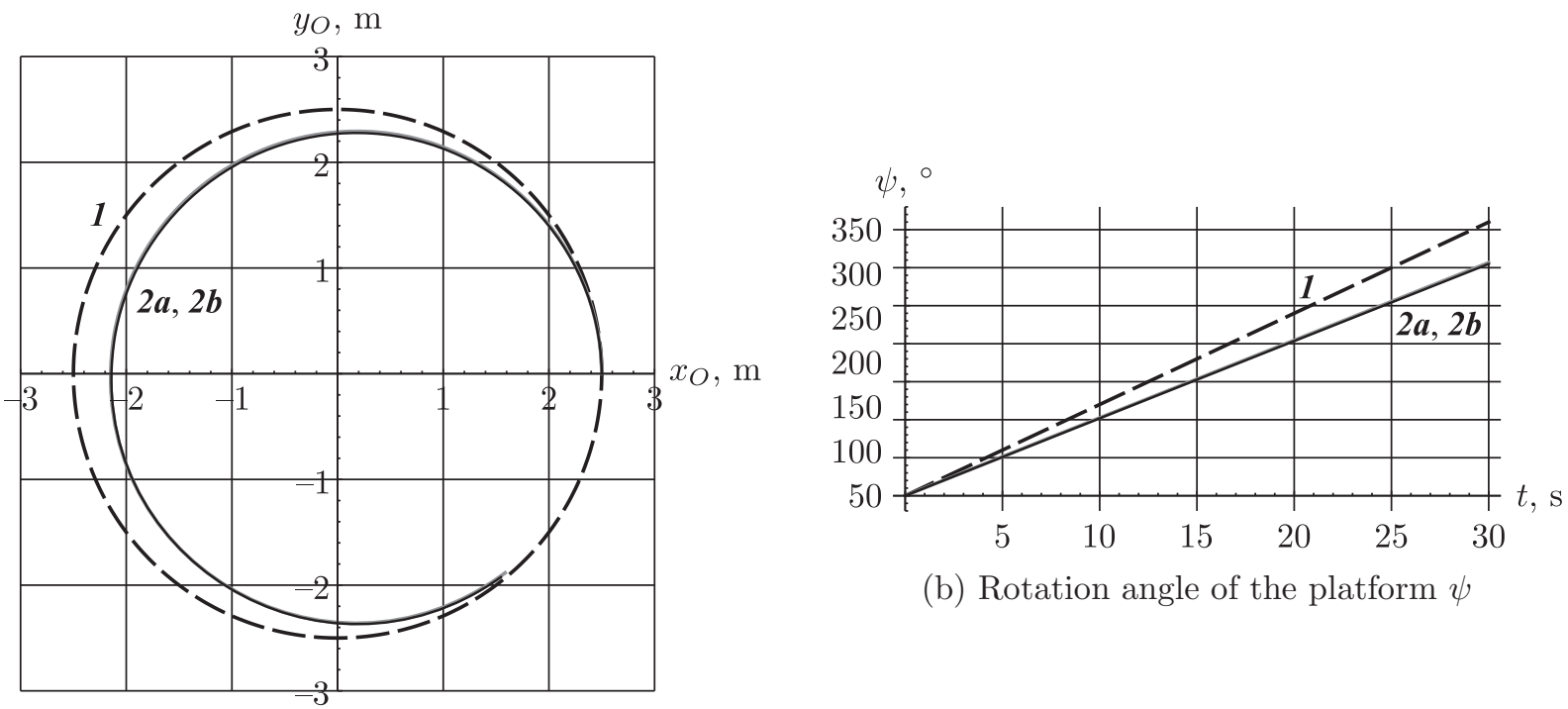

(b) Rotation angle of the platform $\psi$

(a) Trajectories of the center of the platform $O$

Fig. 8. Results of the numerical modeling for Case 2 (the numbers of the curves correspond to those of the models).

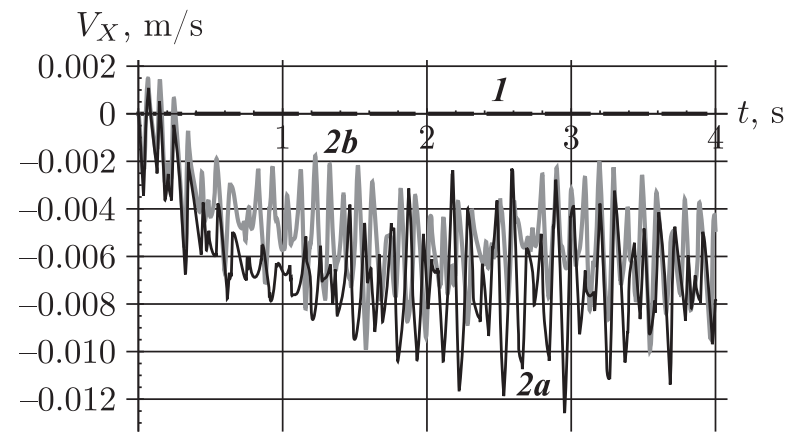

(a) Dependencies $V_{X}(t)$

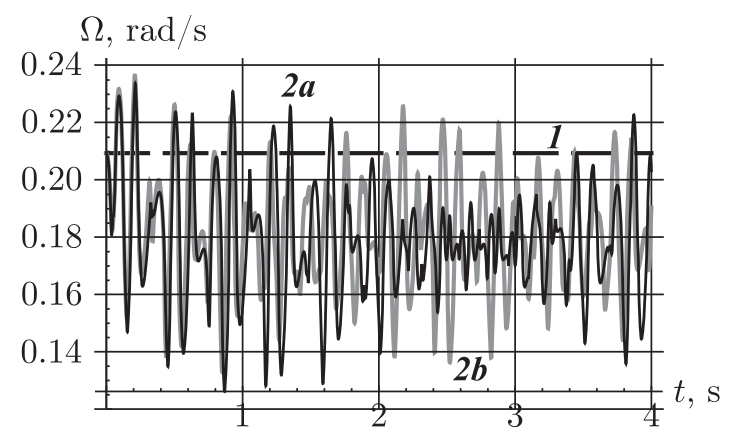

(c) Dependencies $\Omega(t)$

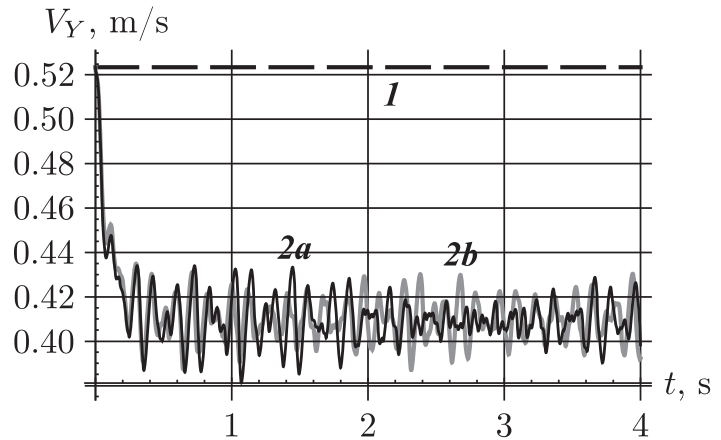

(b) Dependencies $V_{Y}(t)$

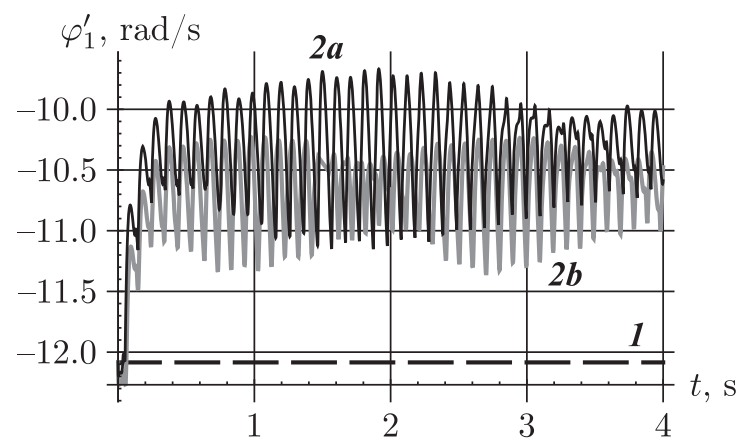

(d) Dependencies $\dot{\varphi}_{1}(t)$

Fig. 9. Results of the numerical modeling for case 2 (the numbers of the curves correspond to those of the models). 
to discontinuous changes in the positions of the contact points $P_{i}$ when switching rollers. In a particular case, according to models $\mathbf{2 a}$ and $\mathbf{2} \mathbf{b}$, the average values of the velocities $V_{Y}$ and $\Omega$ are less than in accordance with model 1. This demonstrates a decrease in the efficiency of the platform's lateral and angular movements control due to the design features of the Mecanum wheels.

Concluding the discussion of the results of numerical simulation, we consider the effects that occur when switching contact rollers. Figure 10 shows graphs of the friction force magnitudes and speeds at the $P_{1}$ point of contact of the first wheel with the reference plane for the initial phase of movement. They demonstrate that when the rollers switch $\left(\varphi_{1}=-\pi / 6\right.$, $-\pi / 2,-5 \pi / 6 \ldots$ ), the linear contact friction forces (model 2a) increase sharply, the Coulomb friction forces (model $\mathbf{2 b}$ ) go into saturation, and the slip rate $\left|v_{P_{1}}\right|$ increases sharply.

Note that after changing the contact roller, $\left|v_{P_{1}}\right|$ decreases to a value comparable to the value of the regularizing parameter $\nu=10^{-3} \mathrm{~m} / \mathrm{s}$.

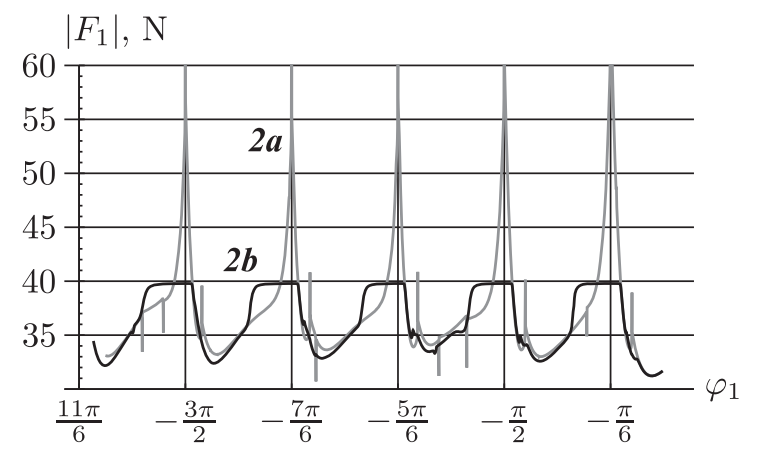

(a) Magnitudes of the contact force $\left|F_{1}\right|$ (the numbers of the curves correspond to those of the models)

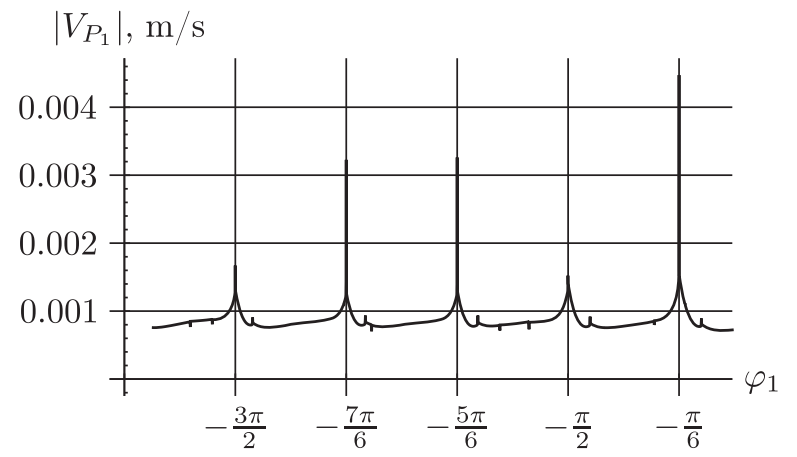

(b) Slip speed $\left|v_{P_{1}}\right|$, Model $2 \mathrm{a}$

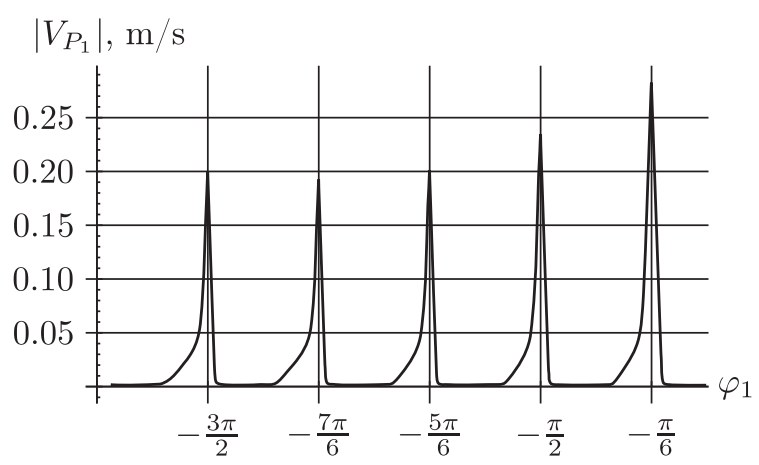

(c) Slip speed $\left|v_{P_{1}}\right|$, Model $2 \mathrm{~b}$

Fig. 10. Forces and velocities at the contact point $P_{1}$ of the first wheel.

\section{Conclusion}

In this paper, the equations of motion of a specific Mecanum platform are derived taking into account the features of the actual design of the wheels and their slippage.

When turning the Mecanum wheel around its axis, the contact point of the roller with the floor is displaced in the direction of the wheel axis. The results of numerical simulation show that this effect leads to a decrease in the efficiency of controlling the angular velocity of the platform 
and the lateral velocity of its center and, as a result, to an increase in energy consumption for the exact implementation of the reference motion and deformation of the trajectories of the platform points.

Switching the contact rollers causes vibrations, which is also illustrated by the results of numerical simulation.

The deformation of trajectories and the dependence of the motion of the Mecanum platform on the initial angle of rotation of the wheels observed in the full-scale experiment [13] can be described using the dynamics models obtained in this paper.

\section{References}

[1] Ilon, B.E., Wheels for a Course Stable Selfpropelling Vehicle Movable in Any Desired Direction on the Ground or Some Other Base: Patent Sweden B60B 19/12 (20060101); B60b 019/00, $\mathrm{REF} / 3876255$ (Nov 13, 1972).

[2] Adascalitei, F. and Doroftei, I., Practical Applications for Mobile Robots Based on Mecanum Wheels: A Systematic Survey, The Romanian Review Precision Mechanics, Optics 85 Mechatronics, 2011, no. 40, pp. 21-29.

[3] Plumpton, J.J., Hayes, M.J. D., Langlois, R. G., and Burlton, B. V., Atlas Motion Platform Mecanum Wheel Jacobian in the Velocity and Static Force Domains, in CCToMM Mechanisms, Machines, and Mechatronics Symposium, 2013, 10 pp.

[4] Ivanov, A.P., On the Control of a Robot Ball Using Two Onmiwheels, Regul. Chaotic Dyn., 2015, vol. 20, no. 4, pp.441-448; see also: Nelin. Dinam., 2015, vol.11, no. 2, pp. 319-327.

[5] Kilin, A. A., Karavaev, Yu. L., and Klekovkin, A. V., Kinematic Control of a High Manoeuvrable Mobile Spherical Robot with Internal Omni-Wheeled Platform, Nelin. Dinam., 2014, vol. 10, no. 1, pp. 113-126 (Russian).

[6] Borisov, A. V., Kilin, A. A., and Mamaev, I.S., An Omni-Wheel Vehicle on a Plane and a Sphere, Nelin. Dinam., 2011, vol. 7, no. 4, pp. 785-801 (Russian).

[7] Martynenko, Yu. G. and Formal'skii, A. M., On the Motion of a Mobile Robot with Roller-Carrying Wheels, J. Comput. Sys. Sc. Int., 2007, vol.46, no.6, pp. 976-983; see also: Izv. Ross. Akad. Nauk. Teor. Sist. Upr., 2007, no. 6, pp. 142-149.

[8] Muir, P. and Neuman, C.P., Kinematic Modeling for Feedback Control of an Omnidirectional Wheeled Mobile Robot, in Proc. of the IEEE Internat. Conf. on Robotics and Automation (Raleigh, N.C., 1987): Vol.4, pp. 1772-1778.

[9] Zobova, A. A., Application of Laconic Forms of the Equations of Motion in the Dynamics of Nonholonomic Mobile Robots, Nelin. Dinam., 2011, vol. 7, no. 4, pp. 771-783 (Russian).

[10] Adamov, B.I., A Study of the Controlled Motion of a Four-Wheeled Mecanum Platform, Russ. J. Nonlinear Dyn., 2018, vol. 14, no. 2, pp. 265-290.

[11] Hendzel, Z. and Rykała, Ł., Description of Kinematics of a Wheeled Mobile Robot with Mecanum Wheels, Modelowanie Inżynierskie, 2015, no. 26(57), pp. 5-12 (Polish).

[12] Xie, L., Scheifele, C., Xu, W., and Stol, K.A., Heavy-Duty Omni-Directional Mecanum-Wheeled Robot for Autonomous Navigation: System Development and Simulation Realization, in IEEE Internat. Conf. on Mechatronics (Nagoya, Japan, 2015), pp. 256-261.

[13] Kilin, A., Bozek, P., Karavaev, Yu., Klekovkin, A., and Shestakov, V., Experimental Investigations of a Highly Maneuverable Mobile Omniwheel Robot, Int. J. Adv. Robot. Syst., 2017, vol. 14, no.6, pp. 1-9.

[14] Gerasimov, K. V. and Zobova, A. A., On the Motion of a Symmetrical Vehicle with Omniwheels with Massive Rollers, Mech. Solids, 2018, vol.53, suppl.2, pp. 32-42; see also: Prikl. Mat. Mekh., 2018, vol. 82 , no. 4, pp. 427-440. 
[15] Moiseev, G.N. and Zobova, A.A., Stability of the Rectilinear Motion of an Omni Vehicle with Consideration of Wheel Roller Inertia, Mosc. Univ. Mech. Bull., 2018, vol.73, no.6, pp. 145-148; see also: Vestn. Mosk. Univ. Ser. 1. Mat. Mekh., 2018, vol.73, no.6, pp. 78-82.

[16] Kosenko, I. I. and Gerasimov, K. V., Physically Oriented Simulation of the Omnivehicle Dynamics, Nelin. Dinam., 2016, vol. 12, no. 2, pp. 251-262 (Russian).

[17] Gerasimov, K. V. and Zobova, A. A., Dynamics of a Vehicle with Omniwheels with Massive Rollers with Account for a Roller Change Contacting with Supporting Plane, Trudy MAI, 2018, no. 101, 26 pp. (Russian).

[18] Gfrerrer, A., Geometry and Kinematics of the Mecanum Wheel, Comput. Aided Geom. Des., 2008, vol. 25, no. 9, pp. 784-791.

[19] Adamov, B. I., Influence of Mecanum Wheels Construction on Accuracy of the Omnidirectional Platform Navigation (on Example of KUKA youBot Robot), in Proc. of the 25th Ann. Saint Petersburg Internat. Conf. on Integrated Navigation Systems, , 2018, pp. 251-254.

[20] http://www.youbot-store.com

[21] Golubev, Yu. F., Appel's Function in the Dynamics of Rigid Body Systems, Preprint No. 49, Moscow: KIAM, 2014 (Russian).

[22] Markeev, A.P., Theoretical Mechanics, Izhevsk: R\&C Dynamics, Institute of Computer Science, 2007 (Russian).

[23] Zimmermann, K., Zeidis, I., and Behn, C., Mechanics of Terrestrial Locomotion: With a Focus on Non-Pedal Motion Systems, London: Springer, 2010.

[24] Butenin, N. V., Introduction to the Analytical Mechanics, Moscow: Nauka, 1971 (Russian).

[25] Adamov, B. I. and Kobrin, A. I., Parametric Identification of the Mathematical Model of the Omnidirectional Mobile Robot KUKA youBot, Mekhatronika, Avtomatizatsiya, Upravlenie, 2018, vol. 19, no. 4, pp. 251-258 (Russian).

[26] http://tehtab.ru/guide/guidephysics/frication/frictiontovariouspairs/ 